\title{
Interaction of Infall and Winds in Young Stellar Objects
}

\author{
G. Delamarter ${ }^{1}$, A. Frank ${ }^{1} \&$ L. Hartmann ${ }^{2}$ \\ 1 Department of Physics and Astronomy, University of Rochester, Rochester, NY 14627 \\ ${ }^{2}$ Harvard-Smithsonian Center for Astrophysics, Cambridge, MA 02138
}

\begin{abstract}
The interaction of a stellar or disk wind with a collapsing environment holds promise for explaining a variety of outflow phenomena observed around young stars. In this paper we present the first simulations of these interactions. The focus here is on exploring how ram pressure balance between wind and ambient gas and post-shock cooling affects the shape of the resulting outflows. In our models we explore the role of ram pressure and cooling by holding the wind speed constant and adjusting the ratio of the inflow mass flux to the wind mass flux $\left(\dot{M}_{a} / \dot{M}_{w}\right)$ Assuming non-spherical cloud collapse, we find that relatively strong winds can carve out wide, conical outflow cavities and that relatively weak winds can be strongly collimated into jet-like structures. If the winds become weak enough, they can be cut off entirely by the infalling environment. We identify discrepancies between results from standard snowplow models and those presented here that have important implications for molecular outflows. We also present mass vs. velocity curves for comparison with observations.
\end{abstract}

Subject headings: ISM: infall and outflows - ISM:molecules - stars: young - stars: winds, wide angle

\section{Introduction}

Molecular outflows are commonly observed in association with young stars. Although the precise mechanism generating the outflows is poorly understood, it is generally believed that the molecular mass is driven and excited into emission by a wind emanating from the inner circumstellar disk close to the star, and driven by accretion energy (Calvet \& Gullbring 1998). Recent observations have explored how these outflows form close to the driving source (Chandler et al. 1996) and how they might evolve in time (Velusamy \& Langer 1998). These 
and other investigations indicate that molecular outflows are not clearly explained in terms of any single current model.

Molecular outflow models are principally divided into two groups; those in which outflows are driven by highly-collimated winds or "jets", and those in which outflows are driven by wide-angle winds. Masson \& Chernin (1992, 1993) and Chernin \& Masson (1995) compared jet driven and wind driven models of molecular outflows. They conclude that jet-driven models are a better fit than wide-angle wind models because observations show very little outflow mass moving at the highest velocities. A jet with moderate post-shock cooling would transfer its momentum to the ambient molecular material largely through the small crosssection of its head, while a wide wind has a much larger driving area. On the other hand, jet-driven models have so far had some difficulty producing the cross-sectional widths of many observed outflows. To reproduce these morphologies, investigators have invoked mechanisms such as turbulent entrainment (Stahler 1994; Raga \& Cabrit 1993), and precession (Cliffe et al. 1995; Suttner et al. 1997). These strategies imply filled outflow cavities and shock structures that aren't generally observed (Chandler et al. 1996). Wide angle wind models can produce wind-blown bubbles with wide bow shocks and empty cavities. It is, however, difficult for the simplest versions of these models to reproduce the observed momentum distribution. Li \& Shu (1996) have suggested that a wide angle wind whose properties vary as a function of polar angle may help solve the momentum problem. Such a wind requires a structure that has higher density along an axis: more like a jet. Thus at present, models and observations leave it unclear as to what is really the driving mechanism of molecular outflows. With these issues in mind, the present paper examines a wide angle wind model through hydrodynamic simulations.

The early model used by Chernin and Masson to make their case against wide angle winds is that presented by Shu et al. (1991). It applies a "snowplow" scenario where a central wind sweeps up ambient material and compresses it into a thin shell. The calculation is performed under the critical assumptions of isothermal shock dynamics and strong mixing between the post-shock wind and ambient gas. The model successfully produces collimated bipolar wind-blown bubbles when relatively denser "equatorial" regions prevent the shell from expanding at low latitudes as quickly as it does along the pole.

New features have been added to the basic wide angle-wind blown bubble model. Close to the young star, effects such as gravity from the central star and the density distribution of the inner envelope become crucial in influencing the shape and evolution of the outflow. This has been investigated in detail by Wilkin \& Stahler (1998), who model a quasi-static balance between a steady central wind, gravity, and a time-dependent angularly varying infall. In essence the bubble is considered as a series of accretion shocks. Using the momentum balance 
across the fixed shell face in both normal and transverse directions, the authors solve for the density and flow pattern within the shell. Because of the quasi-static assumption, the time-dependence of the bubble shape and size is directly linked to the way in which the infall changes in time. The stability of such a situation remains to be investigated. While they found that significant collimation can occur as the environmental density distribution becomes more flattened, the timescale for this is $\sim 10^{5} \mathrm{yr}$. They point out that this is much longer than what is observed. This result reveals the importance of performing dynamic, time-dependent calculations.

While considerable progress has been made in the wide angle wind scenario for molecular outflows there remain many aspects which have not yet been explored. In particular the full time-dependent multi-dimensional nature of the flows has not been examined. Solving cylindrically symmetric non-linear, time-dependent fluid equations by numerical simulation we obtain significantly different behavior from that obtained in previous wide angle wind blown bubble models. The shape of the envelope, namely the gradient of density with polar angle, not only affects the shape of the outer shock and resulting molecular shell, but also the shape of the inner (wind) shock. This can have important dynamical consequences as has been shown in Frank \& Mellema (1996) and Mellema \& Frank (1997).

It seems difficult to avoid the the conclusion that some intrinsic collimation of the initial mass ejection is required, given the evidence for highly-collimated jets over long scales (Bally et al. 1997; Bachiller 1996). This is particularly true for emission-line jets of optically-visible T Tauri stars (e.g., Stapelfeldt et al. 1997; see also the review by Reipurth \& Heathcote 1997), where there is little evidence for the presence of enough external (dusty) medium for initial hydrodynamic collimation of ionized jets to be effective. On the other hand, it should also be recognized that even models of mass ejection with collimated flows often have some wide-angle component. This is true not only of the magnetic "X-wind" model of Shu and collaborators (Shu et al. 1994; Najita \& Shu 1994; Shang et al. 1998), in which a dense axial flow is surrounded by a lower-density dispersing flow; it is also true of the original self-similar MHD disk wind (Blandford \& Payne 1982; Pudritz \& Norman 1983; Königl 1989). For this reason it is worth exploring the interaction of wide-angle flows with the ambient medium.

In this paper we consider the time-dependent interaction of an infalling envelope with an expanding wind. Our focus is spherically-symmetric winds as an example of a maximally wide angle wind, though we do calculate two cases using aspherical winds for comparison. Collimation of the resulting flow will be enhanced if the infalling envelope is not sphericallysymmetric. We take one of the simplest models for such non-spherical collapse, the model of Hartmann et al. (1996), from an initial self-gravitating sheet with no magnetic field.

In $\S 2$ we present the physics and the computational scheme used for the simulations. 
The simulation results are examined in $\S 3$. In $\S 4$ we compare our simulations to the snowplow model of outflows, discuss the possible role and importance of turbulence in these models, and make comparisons of the simulations to observations. We conclude in $\S 5$.

\section{Computation}

\subsection{Methods}

The simulation code solves the Euler ideal fluid equations with source (sink) terms for central point-source gravity and radiative cooling. The equations, written in conservative form, are:

$$
\begin{array}{r}
\frac{\partial \rho}{\partial t}+\nabla \cdot \rho \mathbf{u}=0, \\
\frac{\partial \rho \mathbf{u}}{\partial t}+\nabla \cdot \rho \mathbf{u} \mathbf{u}=\rho \nabla \Phi \\
\frac{\partial \epsilon}{\partial t}+\nabla \cdot \mathbf{u}(\epsilon+p)=-\Lambda(\rho, T)-\rho \mathbf{u} \cdot \nabla \Phi
\end{array}
$$

where $\rho$ is the fluid mass density at a point, and $\mathbf{u}$ is the velocity. The pressure $p$ is related to the energy density $\epsilon$ and its kinetic $\left(\epsilon_{k n}\right)$ and thermal components $\left(\epsilon_{t h}\right)$ through the relations

$$
\begin{gathered}
\epsilon=\epsilon_{k n}+\epsilon_{t h}, \\
\epsilon_{k n}=\frac{1}{2} \rho u^{2}, \\
\epsilon_{t h}=\frac{p}{(\gamma-1)} .
\end{gathered}
$$

The adiabatic index $\gamma=5 / 3$ is that for a monatomic gas. The temperature is determined through the ideal gas equation of state, with the particle mass set to that of atomic hydrogen:

$$
p=\frac{\rho k T}{m_{H}} .
$$

The cooling source term $\Lambda(\rho, T)$ includes a Dalgarno-McCray 1972 cooling curve which is applied above $6000 \mathrm{~K}$, and a Lepp and Shull 1983 cooling curve applied below $6000 \mathrm{~K}$ to simulate the effects of both high and low temperature cooling.

Gravity in these models is purely due to a source at the origin. Self-gravity of the fluid is not included. The gravity source term is written in terms of the gradient of the potential 
$\nabla \Phi$. This force is specified via the constant central source mass $M_{*}$ as

$$
\nabla \Phi=G \frac{M_{*}}{r^{2}} \hat{r}
$$

The radius $\mathbf{r}$ is the vector from the origin to the fluid point.

The equations are solved on an Eulerian grid using an operator split numerical method, where several operators are applied per timestep, each one simulating a different aspect of the physics. We also employ fluid tracking to determine what part of the initial grid a fluid parcel came from. The grid covers a quarter meridional plane of a cylinder and has assumed axial symmetry plus reflective symmetry across an equatorial plane. The basic hydrodynamics equations without source terms are applied via the numerical Total Variation Diminishing (TVD) method of Harten 1983 as implemented in dimensionally split form by Ryu et al. (1995). The source term for radiative cooling is applied explicitly to first order via an exponential as described in Mellema \& Frank (1997) where:

$$
\epsilon_{t h}^{n+1}=\epsilon_{t h}^{n} \exp \left(-\frac{\Lambda^{n}\left(\rho^{n}, T^{n}\right)}{\epsilon_{t h}^{n}} \Delta t\right) .
$$

The superscripts represent a value at the $n$th and $(n+1)$ th timestep, and $\Delta t$ represents the amount of time between timestep $n$ and $n+1$. Gravity is applied through a first order explicit Euler operator which updates the velocities and the kinetic energy density. The fluid is evolved by rotational velocity through another first order explicit Euler operator.

The timesteps are adjusted to satisfy the Courant condition on the sourceless hydrodynamics, and simultaneously to be less than a reasonable multiple of the cooling time: $3 \Lambda(\rho, T) / \epsilon_{t h}$. Because of the high densities in the ambient medium $\left(n>10^{8} \mathrm{~cm}^{-3}\right)$ the cooling time step can become relatively small and the number of times steps required to complete the simulation relatively large. This produces additional diffusion in the solutions causing our flows to appear rather smooth. By artificially reducing the cooling in some test simulations, we were able to obtain sharper features. The gross morphology and evolution of the flow however remained qualitatively the same as those with the full cooling shown in $\S 3$.

We applied several tests to the code. We compared momentum conserving wind blown bubbles with the solutions obtained by Koo \& McKee (1992) to test the basic hydrodynamics with strong cooling. We performed radiative shock tube tests along the axis using a simplified power law cooling function and compared these against semi-analytic shock solutions to test the cooling operator. To test gravity we reproduced the Bondi accretion solution in the code. To test gravity and rotational velocity, we maintained a Keplerian ring in an orbit around a central mass. In general, the code was able to recover the analytical solutions to better than $10 \%$. 


\subsection{Model}

The scenario we applied involved driving a spherically symmetric wind into an infalling non-spherical envelope. While a spherical wind may not be operating in many young stellar object systems, we seek in this investigation to explore the shaping of the outflow through wind/environment interactions and make contact with previous analytic studies. We hope to relax the assumption of a spherical wind in a later paper. The parameters used are given in Table 1 and are based on typical values for low mass young stellar objects. A radially directed steady wind of velocity $200 \mathrm{~km} / \mathrm{s}$ and mass flux $10^{-7} M_{\odot} / y r$ was imposed at each timestep on the cells of the grid within an origin-centered sphere of radius equal to $10 \%$ the radial size of the simulation. We chose an environment given by Hartmann et al. (1996) derived from their simulations of a collapsing, rotating, axially symmetric sheet. In this model, the collapse of the initially-flattened protostellar cloud gives rise to a highly anisotropic density distribution in the infalling envelope, which in turn helps strongly collimate the initially spherical flow. As Hartmann et al. point out, whether or not this model is correct in detail, the general property of non-spherical clouds to flatten or become more anisotropic as gravitational collapse proceeds lends credence to the idea of aspherical infall. The anisotropic stresses of magnetic fields can also produce non-spherical protostellar clouds, and Li \& Shu (1996) argue that such initial configurations can also help make outflows more collimated. ${ }^{1}$

The density is given by the equations (8), (9) and (10) in Hartmann et al. (1996) which modifies calculations by Cassen \& Moosman (1981) and Terebey et al. (1984) (referred to hereafter as TSC). The velocity distribution is taken from Ulrich (1976). The equations are rewritten in a slightly different but equivalent form in the appendix. These equations produce a flattened infalling toroidal density distribution with an equator to pole density contrast $\rho_{e} / \rho_{p}>1000$. This is larger than what is produced by the Cassen \& Moosman and TSC models. The TSC model was used by Wilkin \& Stahler (1998).

The environment near the center of the sheet models used in this paper is a torus of a high equatorial density $\left(6 \times 10^{8} \mathrm{~m}_{H} / \mathrm{cm}^{3}\right)$ with a half-maximum opening angle of almost 180 degrees. In the hydrodynamic collimation simulations of Frank \& Mellema and Mellema \& Frank a "fat" torus was used with opening angles of $\sim 90^{\circ}$, similar to those obtained by Li \& Shu (1996) for magnetized collapse. These previous simulations produced outflows with strong collimation. It is noteworthy that, as we shall see in $\S 3$, it is possible to produce

\footnotetext{
${ }^{1}$ However, we note that the static configurations suggested by Li \& Shu may not be particularly relevant to outflow sources, because some collapse must have already occurred to produce the central mass responsible for the outflow. As shown by Hartmann et al. (1994), even if the initial density configuration is not toroidal, or magnetically-dominated, a toroidal density distribution will result from collapse.
} 
strongly collimated flows even with the wide opening angles of the sheet distribution.

We investigate the influence of ram pressure balance between wind and ambient gas by holding the wind speed constant and varying the ratio of the inflow mass flux to the wind mass flux (denoted $f^{\prime}$ :)

$$
f^{\prime}=\frac{\dot{M}_{i}}{\dot{M}_{w}} .
$$

We chose to hold the wind velocity constant because young stellar object wind and jet velocities are well observed to be a few hundred kilometers per second (Bachiller 1996; Shu et al. 1991), while the range of the mass flux ratio f' is less well known. We have focused on models with $f^{\prime}=10,20,30,40$, and 50 . This was accomplished by adjusting the infall mass flux and fixing the outflow mass flux. We also simulated cases where the inflow mass flux was reduced relative to the wind, and obtained basically the same results with only minor differences. We note that a measure of the effect of varying $f^{\prime}$ can be seen by calculating the angle $\theta_{e}$ at which the ram pressures are equal in our simulation. For $f^{\prime}=10$ we find $\theta_{e}=1.568$ radians (measured from the axis) which is less than $6 \%$ of a grid cell. For $f^{\prime}=50$ we find $\theta_{e}=1.519$ radians which is less than $130 \%$ of a grid cell. Thus is is clear that the wind will be able to push all material away from the equator in the $f^{\prime}=10$ case while $f^{\prime}=50$ simulations should experience some material attempting to cross the inner wind sphere.

We note here the basis of our attack on this problem. The theoretical issue of the interaction of a stellar wind with an infalling environment is complex. There are a number of parameters controlling both the wind and the infalling environment. In choosing to address the issues involved one must ask which parameters are important enough, a-priori, to warrant attention? Which connect directly to issues raised in previous studies? Which allow for a numerically clean solution, i.e. one whose specification of initial and boundary conditions do not impose serious transients or artifacts on the flow? In taking on this problem we have adopted a strategy which, hopefully, allows to us address important aspects of the physics while leaving others for future works. In the present paper we focus primarily on a relatively simple treatment of initial/ boundary conditions (e.g. the wind and infalling environment) in order to clarify how a full solution to the governing equations differs from the more idealized solutions explored in previous works. We have constructed a focused exploration of a set of numerical experiments that address key issues not explored in previous studies. In future works we plan to open other dimension of the parameter space including a more detailed treatment of aspherical winds. 


\section{Results}

\subsection{Spherical Winds/Aspherical Environments}

The sampling in $f^{\prime}$ described in the previous section can be thought of as a sampling from a relatively strong wind to a relatively weak wind, as the results will show. In addition to exploring different steady wind cases, the simulations serve as a crude initial exploration of what might be expected in models with fully time-dependent wind mass loss rates. The details of time-dependence are particularly important for FU Orionis stars (Hartmann \& Kenyon 1996) where the observations show considerable variation in the wind, likely related to large fluctuations in the accretion rate of material through the disk. In this section, we start with a general overview of the results and continue by considering each simulation in more detail.

The principal results can be inferred from Fig. 1 showing density snapshots of all five simulations. In the $f^{\prime}=10$ case we see the strong wind overwhelms the infall and creates a wide conical cavity. As $f^{\prime}$ decreases, the wind becomes weaker, the infall mass flux dominates and, as a result, the wind becomes more confined and collimated. Even in the $f^{\prime}=50$ case however the wind is still strong enough to avoid being completely cut off. In simulations where we increase the infall flux such that $f^{\prime}=100$, the wind is completely choked by the infall with its momentum slowly diffusing into the surrounding environment. Because of the diffusion and the direct interaction of the infall with the artificial wind boundary, we are not confident in the simulations after the wind becomes choked. Therefore we have only placed a lower limit $\left(f^{\prime}=50\right)$ on the infall to wind flux ratio at which the wind is cut off.

In the strong wind, $\left(f^{\prime}=10\right)$, case (see Fig. 2), the wind pushes its way through the environment but is still shaped by it. By the time the swept up ambient material reaches the top of the grid (at $\sim 180 y$ : see Figs. 3 and 4) it has a conical shape reminiscent of the outflows seen in Velusamy \& Langer (1998) and Chandler et al. (1996). The outflow bubble opens with an angle of about $30^{\circ}$ to the vertical. The supersonic wind and molecular material are each compressed at the inner and ambient shocks respectively, forming a thin layer about the contact discontinuity at $20^{\circ}$ to the vertical. The dynamics of this region are dominated by the fact that the radially directed wind material hits the inner shock obliquely. Post-shock wind is redirected and forced to flow along the contact discontinuity. Eventually this high speed gas flows up the walls of the conical cavity and reaches a cusp at top of the bubble. There the focused high speed flow moves ahead of the bubble, encircling its

nearly spherical cap with a conical "jet". While the development of such a pattern is readily understood in terms of basic radiative oblique shock physics it is not clear that the cusps and resulting jets would be stable in real three dimensional bubbles. 
Table 1. Model parameters.

\begin{tabular}{cc}
\hline \hline parameter & value \\
\hline Simulation Domain & $4.26 \times 10^{15} \mathrm{~cm}$ radius by \\
& $8.52 \times 10^{15} \mathrm{~cm}$ along axis \\
256 cells radially by \\
Resolution & 512 cells along axis \\
Wind velocity, $v_{w}$ & $200 \mathrm{~km} / \mathrm{s}$ \\
Wind mass flux, $\dot{M}_{w}$ & $10^{-7} M_{\odot} / y r$ \\
Central mass, $M_{*}$ & $0.21 M_{\odot}$ \\
Infall mass flux, $\dot{M}_{a}$ & $10^{-6} M_{\odot} / y r \times$ \\
Collapse radius, $r_{0}$ & $5.37 \times 10^{16} \mathrm{~cm}$ \\
Flattening parameter, $\eta$ & 2.5 \\
Centrifugal radius, $R_{c}$ & $4.28 \times 10^{14} \mathrm{~cm}=28.5 \mathrm{AU}$ \\
\hline
\end{tabular}

Fig. 1.- Comparison between bubbles at times of similar axial extent. The density of the $f^{\prime}=10,20,30,40$, and 50 (left to right - strong wind to weak wind) cases are shown at their full heights at 100, 200, 220, 240, and 220 years, respectively. The inner wind boundary makes a quarter circle around the origin with radius $4.26 \times 10^{14} \mathrm{~cm}$. Note the change in aspect ratio of the bubbles as the wind becomes weaker. 
It is interesting that as the bubble evolves, a region of warm gas $\left(T \approx 10^{5} \mathrm{~K}\right)$ develops at the top pushing the inner and outer shocks apart there. Usually wind blown bubbles are classified as either adiabatic or radiative depending on whether the cooling time is longer or shorter than the expansion time of the bubble. We believe that this top region does not fall into either category, and that instead we are seeing the development of a partially radiative bubble. This third classification, which lies between the adiabatic and radiative cases, was originally explored by Koo \& McKee (1992). Such a case occurs when the wind material resupplies thermal energy generated at the shock faster than radiative cooling can remove it, but when the cooling time is still shorter than the bubble expansion time. To see if the system is partially radiative at the top of the $f^{\prime}=10$ bubble we need to compare the cooling time $t_{c}$ with the wind crossing time $t_{x}$.

Given that the top of the bubble has an essentially spherical shape we can use the analysis of 1-D bubbles to write the cooling and crossing times in terms of the distance from the wind source, $R$, plus other simulation parameters for the $f^{\prime}=10$ case. A rough estimate of the inner shock speed is $v_{s}=v_{w}=200 \mathrm{~km} / \mathrm{s}$ which produces a temperature of $8.8 \times 10^{5} \mathrm{~K}$. The Dalgarno-McCray cooling curve at this temperature gives a $\Lambda(n, T) / n^{2}$ of $1.65 \times 10^{-22} \mathrm{erg} \mathrm{cm} / \mathrm{s}$. Using $\dot{M}_{w}=10^{-7} M_{\odot} / y r$ and the factor of 4 density increase behind a strong shock we can determine the cooling time from the following equation:

$$
t_{c}=\epsilon_{t h} / \Lambda(n, T) \sim 4100 s(R / A U)^{2}
$$

The crossing time, $t_{x}$, is given simply in terms of the radial distance from the origin to the shell and the wind velocity:

$$
t_{x}=\frac{R}{v_{w}} \sim 7.5 \times 10^{5} s(R / A U)
$$

Note that the crossing time grows linearly and the cooling time grows quadratically. Thus the cooling time eventually surpasses the crossing time. For the parameters given above this occurs when the shock is at a distance of about $182 \mathrm{AU}$ or $2.7 \times 10^{15} \mathrm{~cm}$ which is well inside the simulation domain. We find that this is approximately the distance where the simulated bubble begins to exhibit its cap of warm postshock wind material. We believe that this is the first time that the partially radiative bubble phase has been seen in a simulation, confirming the prediction of Koo \& McKee.

The weaker $f^{\prime}=20$ case is seen in Fig. 1 to also develop a conical outer shock, but the overall shape is more confined or collimated than in the previous case. The cusp which forms at the edge of the bubble is more pronounced. This effect may due to higher inertia of the 
Fig. 2.- The density evolution of the $f^{\prime}=10$ (strong wind) case. The bubble begins spherical but breaks out into a cone. Other features such as a partially radiative shock and conical "jets" appear (see text).

shocked wind flow. At low latitudes material is channeled from regions closer to the origin where the density is higher. In addition, the polar regions of the bubble are expanding slower due to the relatively lower wind mass loss rate. Thus the refracted wind material emerges from the top of the bubble with higher momentum relative to the polar cap in this model than in the $f^{\prime}=10$ case, allowing the cusp to propagate farther from the cap. Another difference at the pole is that less warm post-shock gas exists in this case. This effect can also be attributed to the the slower speed of the polar sectors of the bubble. A slower outer shock speed implies a faster inner shock speed (in the frame of the wind). This in turn leads to higher post-shock temperatures and stronger cooling in the shocked wind. Note that the inner shock forms an angle of about $10^{\circ}$ to vertical and the outer shock (bubble) opens roughly to $20^{\circ}$.

At weaker winds $\left(f^{\prime}=30\right)$ the inner shock becomes more confined and the flow is even more collimated. The cusp and resulting conical "jet" is more pronounced than seen previously. Again this is expected because the cap of the bubble will expand at lower velocities. Figure 5 shows the details of the flow pattern for this case. Since the $f^{\prime}=20$ and $f^{\prime}=30$ models are similar the figure emphasizes the points concerning the flow pattern made above. The wind shock opens about $2^{\circ}$ to vertical and the bubble opens about $20^{\circ}$.

In the second weakest case $\left(f^{\prime}=40\right)$ the inner shock becomes almost elliptical or 


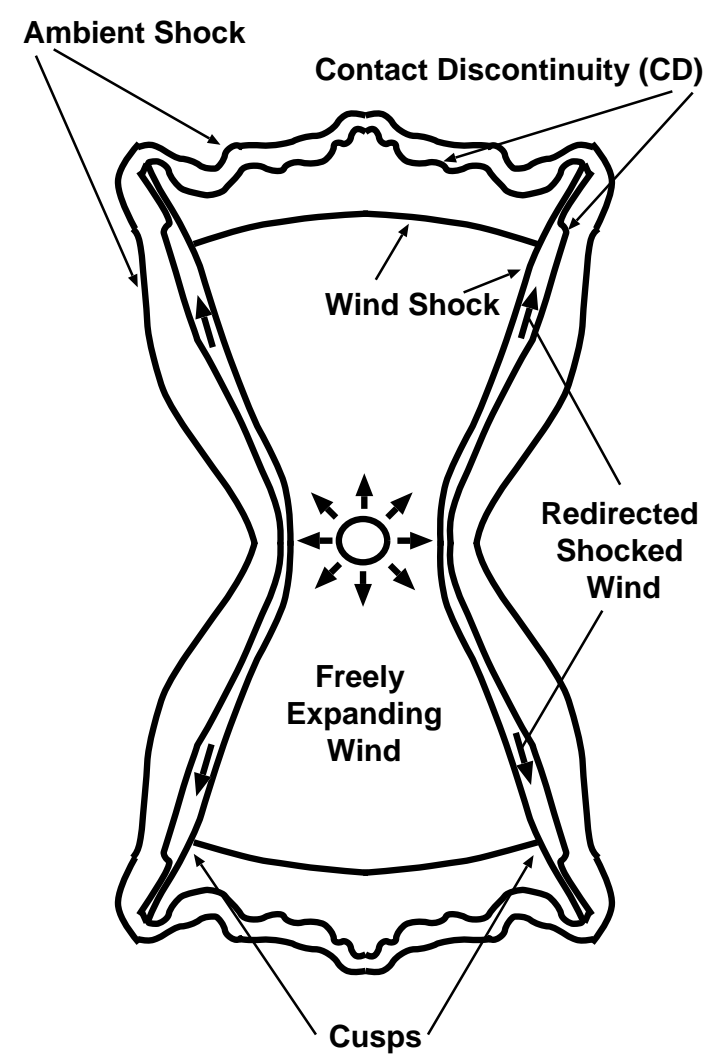

Fig. 3. - A cartoon of the $f^{\prime}=10$ (strong wind) case, labeling the location of the important fluid-dynamics features mentioned in the text. The central source and freely expanding wind are shown. The major surfaces of discontinuity (shock, contact) are identified as well as the sharp cusps in the wind shock. The flow of the redirected wind is shown schematically. 
Fig. 4.- The density, pressure, temperature, and velocity of the $f^{\prime}=10$ (strong wind) case at 180 years. The velocity magnitude is represented by grayscale shading and the direction is represented by overlaid normalized arrows. Note that in the freely expanding wind region (white) the velocity vectors have been sampled at regular grid points and fool the eye into seeing a precollimated wind. The wind in that region is actually expanding spherically. 
Fig. 5.- The density, pressure, temperature, and velocity of the $f^{\prime}=30$ (moderate wind) case at 220 years. 
Fig. 6. - The density evolution of the $f^{\prime}=50$ (weak wind) case. Confinement is strong, and is maintained by a prolate shock.

"bullet" shaped. The tip of the shock at the axis is suspect because of the imposition of reflective boundary conditions and may not form if the cylindrical symmetry is relaxed (see for example Stone \& Norman 1994). The wind is strongly focused by the oblique inner shock producing an almost vertically directed high velocity flow. The resulting bubble is very well collimated. In this case the conical cusp is not as pronounced and the dynamics look very much like a jet. At the tip of the outflow (along the axis) the the inner shock is acting in the same manner as a jet shock, decelerating vertically directed wind and shunting it off in the direction transverse to the propagation of the outflow. The full opening angle of the outer shock of the bubble is about $35^{\circ}\left(17.5^{\circ}\right.$ to vertical).

The weakest wind case $\left(f^{\prime}=50\right)$ is shown in Figs. 6, 7. It exhibits very strong collimation. The inner shock is strongly prolate and closes back on itself at a relatively small distance from the central source, and redirects all the wind into the collimated outflow. There is no extended region of freely expanding wind. This occurs because inflowing material is overwhelming the wind at low latitudes, inhibiting its expansion everywhere except at the poles. Even though the opening angle of the bubble's outer shock at the base is $35^{\circ}$, the resulting outflow will appear cylindrical because of the focusing.

The trend of the evolutionary timescales of the different simulations allows us to infer the dynamical consequences of collimation. The timescales are taken from the snapshots in Fig. 1 which were chosen such that the bubbles are of comparable height. As the wind 
Fig. 7.- The density, pressure, temperature, and velocity of the $f^{\prime}=50$ (weak wind) case at 220 years. Note the focusing effect of the inner shock evident in the velocity plot, and the wide region of vertically directed flow. 
becomes weaker $\left(f^{\prime}=10\right.$ to $\left.f^{\prime}=50\right)$ the timescales go $100 y, 200 y, 220 y, 240 y, 220 y$. The bubbles decelerate as the wind weakens. The effect saturates however and then reverses as the collimation becomes stronger. At first glance this reversal may be unexpected because as winds become weaker they should supply less momentum to the bubble. However, as the wind becomes focused into a jet the net flux of momentum, $F=\rho v^{2} A$, is injected into the environment across a smaller angular extent $A$. Thus the tip of a highly collimated bubble expands at a higher velocity than would occur for a spherical bubble.

We shifted from the "strong wind" to "weak wind" cases in these simulations by increasing the infall mass flux while fixing the wind velocity. If instead we shift from $f^{\prime}=10$ to the $f^{\prime}=50$ by decreasing the mass flux of the wind and keeping the infall mass flux fixed we find that the results are qualitatively the same. The post inner shock region expands slightly faster due to the lower density and reduced cooling there, but the effect is not significant. The results are, therefore, determined solely by the parameter $f^{\prime}$.

The results of the simulations show the complicated way in which the environment shapes the outflow. This occurs directly at the outer shock by providing an inertial gradient along the shock face. It also occurs indirectly by affecting the shape of the inner shock. Shaping the inner shock leads to shock focusing of wind material, creates cusps at the tips of the bubble and, in the most extreme cases, leads to focusing into a jet through prolate inner shocks. In all cases, the simulations show the "internal" flow of post inner shock wind material plays an important role in the dynamics.

The results also show the way in which outflows affect the environment. The winds carve out evacuated cavities of potentially large angular extent and sweep ambient material at a high rate. The swept up mass totals $1 \times 10^{-6} M_{\odot} / y r$ to a few times $10^{-7} M_{\odot} / y r$ as the wind becomes weaker. Because the winds become more collimated and on the whole expand much more slowly as they weaken, ambient mass is swept at a slower rate. The wider angle winds are able to collect mass much more rapidly even though the envelope has less material in it.

\subsection{Spherical Winds/Aspherical Environments}

Although they are not the focus of this study, we have included the results of simulations with aspherical winds injected into a spherical environment for comparison. We note that addressing the issue of aspherical winds means specifying a model for those winds. This raised a number of questions suh as; should simple sinusoidal variation be used or something akin to the cylindrical stratification seen in wide-angle models like those of the X-winds? If 
we focus on the former should we vary only the density from pole to equator or only the velocity. If they both vary then should momentum or energy flux be held constant across the stellar surface? Given these the complexities we have chosen a highly simplified set of boundary conditions for these models and present them as means of contrasting the results of the previous section.

The distribution in the environment is given by Terebey et al. (1984) with the same infall parameters as used in the strong-wind simulation described above: essentially it is the previously described sheet distribution except unflattened. The wind velocity distribution is given by

$$
v_{w}(\cos \theta)^{\log \left(\cos \theta_{c}\right) / \log \frac{1}{2}}
$$

where $\theta$ is the polar angle, $v_{w}$ is the maximum polar $200 \mathrm{~km} / \mathrm{s}$ velocity and the angle of half-maximum velocity is $\theta_{c}$. The wind mass density at every point is kept the same as in the strong-wind simulation.

The density of the result of injecting a mildly focused wind $\left(\theta_{c}=70^{\circ}\right)$ is shown in Figure 8. Even at 300 years, the bubble is much more limited in size than in the sheet environment cases: the expansion speed is lower and very little warm gas exists to push the bubble outwards. The focusing is also much more limited because of the the environment.

The resulting density snapshot of injecting a more focused wind $\left(\theta_{c}=30^{\circ}\right)$ into the spherical environment is shown in Figure 9. Here the focusing is pronounced, but the bubble extent at 300 years is still comparatively small because of the greater mass in the polar regions. As is to be expected, a focused wind in a spherical environment can also produce a focused outflow. Detailed results, however, such as the precise bubble shape should not be taken as final because the infall material is able to press up against the artificial wind boundary.

These simulations show that the shape of a wind blown bubble is not unique to a single set of boundary conditions. As one might expect we do see collimated outflows emerging from collimated winds though there are some differences from the spherical wind case in terms of propagation speed and post-shock temperatures. These results show that the issue is always one of wind momentum or ram pressure injection as a function of angle. With sphericalwinds/aspherical-infall shock focusing helps redirect the wind ram pressure towards the pole. This is explored in more detail in the next section. In the aspherical winds/spherical-infall case the wind momentum must be strongly collimated a-priori to produce a narrow outflow.

Thus flow shapes are not unique nor should one expect them to be. However, as we will explore in the next section, the simulations presented in section 3.1 demonstrate an 
Fig. 8. - The density at 300 years of a case where a non-spherical wind is injected into an isotropic infalling environment. The half-maximum velocity is at $\theta_{c}=70^{\circ}$ from the pole. Note the small extent of the bubble at this late time and the minor focusing.

effectiveness of wide-angle wind models not seen in previous studies.

\section{Discussion}

In this section we discuss the results of our simulations in light of previous analytical models of YSO wind blown bubbles We also attempt to establish some points of contact between our models and molecular outflow observations. In making these comparisons we must note a limitation of the present simulations, namely that they formally refer to only very small spatial and temporal scales. The overwhelming majority of observed molecular outflow structures have typical lifetimes of $10^{4}-10^{5} \mathrm{yr}$, in contrast to the $10^{2} \mathrm{yr}$ time sequences shown here. In addition, for flows that are much larger than $0.1 \mathrm{pc}$, it is very likely that the bulk of the swept-up interstellar material originally resided in regions outside the protostellar core, let alone the inner infall regions. Nevertheless, there are a couple of observations of younger and smaller outflows (Chandler et al. 1996) where our simulations may be more directly relevant. In addition some of the physical results may be generalized 
Fig. 9. - The density at 300 years of a case where a narrow wind is injected into an isotropic infalling environment. The half-maximum velocity is at $\theta_{c}=30^{\circ}$ from the pole. The outflow is collimated as expected. Here, as in the wider wind case in Figure 8, we find that the bubble is growing much more slowly than those formed in the collapsing sheet environment. The infalling material is reaching the wind sphere in this case, so the exact shape of the bubble obtained in this simulation is suspect.

to larger-scale situations.

\subsection{Comments on the Shu, et. al. "Snowplow" Outflow Model}

In Shu et al. (1991) a simple, elegant "snowplow" model of the formation of bipolar molecular outflows is presented. The basic features of this model were a central wind with a mass flux varying with polar angle and an environment with a density that varies with polar angle and falls off in radius as $1 / r^{2}$. A number of simplifying assumptions are invoked. Cooling is assumed instantaneous so that the bubble is a purely momentum-driven thin shell. The interaction of the ambient material and the wind is taken to be fully mixed and ballistic such that the dynamics at a given polar angle are independent of those at nearby angles. In 
addition, the swept up wind mass is taken to be negligible.

In the snowplow model, changing the ratio of total infall to wind mass flux $\left(f^{\prime}\right)$ only effects the bubble's expansion timescale but doesn't change the self-similar shape. The $1 / r^{2}$ ambient profile leads to a simple expression for the bubble shape and the variation with polar angle in the extent of the bubble is purely determined by the angular variations of wind and

ambient medium. The simulations however show a strong variation of bubble shape with $f^{\prime}$. This discrepancy implies that some dynamical aspects of the simulations are not captured in the snowplow model.

The most important physical difference between the simulations and the snowplow model is that the snowplow model does not account for the post inner shock redirection of wind material. Pressure gradients in the post-shock shell that come about because of the variation in the ambient density, and resulting variations in shock obliquity and shock speed have no consequence in the snowplow model, but have profound effect in the simulations. If we were to somehow model the non-radial direction of the postshock wind and use that in the snowplow model we might obtain a better agreement. The question becomes should one use the flow inside or outside the inner wind shock as the input wind to the snowplow model. While this may seem, on one level, to be a question of where one starts the calculation, more fundamentally it is an issue of what initial physics one includes to determine the wind distribution. Li \& Shu (1996) make a modification of the snowplow model in this way by incorporating the distribution of the wind after it has been accelerated and partially focused by magnetic launching.

If dynamically important physics in wide wind scenarios are left out of the snowplow model, it leads to the question whether the model is useful in predicting observations. Masson \& Chernin (1992) and Li \& Shu (1996) have used the snowplow model to calculate distributions of mass and velocity to argue whether or not wide angle winds can drive outflows. Our simulations, however, show that even if the driver is initially a wide angle wind it can be focused by a nonlinear interaction between the wind and environment. Our simulations appear to blur the distinction between wide angle winds and jets as the sole driving mechanism of the outflow.

\subsection{Mixing and momentum transfer}

A strong assumption used in the analytic models of Shu et al. (1991) and Wilkin \& Stahler (1998) is that postshock wind and postshock ambient material become fully mixed on a dynamically short timescale. This allows the shell to be treated as a single fluid and 
greatly simplifies the calculations. To support the assumption, Wilkin \& Stahler consider supersonic shear flows within the postshock regions and state that some form of instability will generate turbulence and rapidly mix the wind and ambient fluids. Our simulations have some diffusive mixing of fluids due to numerical effects, but no complete mixing occurs and there is no turbulence modeled in the code. Since the simulations show the post-shock shear flow influencing the shape of the bubble, it is worth discussing when turbulent mixing of ambient and wind material will occur and, if it does occur, how it might affect the bubble's evolution.

\subsubsection{Turbulent length scale}

The onset of turbulence in jets and bubbles is still poorly understood, but one estimate of the momentum transfer rate between material in a jet and a stationary ambient medium has been performed by Cantó \& Raga (1991). From this, they calculate the length, $L_{t}$, a laminar jet can travel before it is subsumed in a turbulent boundary layer finding that $L_{t}$ scales linearly with jet Mach number, and inversely with the jet radius $R_{j}$. At Mach 10 they find $L_{t} / R_{j} \approx 170$. The calculations were performed assuming slab symmetry and are applicable to our supersonic postshock wind regions. Raga et al. (1995) extend the results to the case where both fluids are moving and find that the spreading rate is actually reduced.

Using the result of Cantó and Raga we can estimate when the flows inside the bubble become turbulent. For this purpose we calculate the width of the shell of post-shock wind in a slightly aspherical isothermal wind-blown bubble. The calculation is simplified by the nearly spherical shape, but is still consistent with shock focusing because even mildly aspherical bubbles will produce focused flows (Frank \& Mellema 1996). If density $\rho_{w s}$ in the postshock region is constant, the mass in that region, $M_{w s}$, can be written in terms of the volume $V_{w s}$ as:

$$
M_{w s}=V_{w s} \rho_{w s}
$$

If the speed of the shock $v_{s}$ is small compared with that of the wind, $M_{w s}$ can also be written

$$
M_{w s}=\dot{M}_{w} t
$$

where $\dot{M}_{w}$ is the mass flux of the wind and $t$ is the age of the bubble. If the shock is relatively thin and the bubble is not too aspherical then we can approximate the volume as $V_{w s} \sim 4 \pi R_{s} \Delta R_{w s}$, where $R_{s}$ is the shock radius and $\Delta R_{w s}$ is the shock width. In terms 
of the shock Mach number $\tilde{M}$ and the preshock wind density $\rho_{w}$ the isothermal condition requires that $\rho_{w s}=\rho_{w} \tilde{M}^{2}=\tilde{M}^{2} \dot{M}_{w} /\left(4 \pi R_{s}^{2} v_{w}\right)$ (Shu 1992). Equating (14) and (15) and solving for the shock width gives:

$$
\Delta R_{w s}=\frac{t v_{w}}{\tilde{M}^{2}}
$$

Once we calculate a shock speed, we can calculate the relative width of the bubble, $\Delta R_{w s} / R_{s}$. For simplicity we choose the ambient environment to fall as $1 / r^{2}$, which, as mentioned in the previous section, implies a constant shock velocity of $v_{s}=\sqrt{\left(\dot{M}_{w} / \dot{M}_{a}\right) v_{w}(a / 2)}$. Choosing typical values of $v_{w}=100 \mathrm{~km} / \mathrm{s}, \dot{M}_{w} / \dot{M}_{a}=1 / 10, \tilde{M}=100, a=0.2 \mathrm{~km} / \mathrm{s}$, we obtain:

$$
\frac{\Delta R_{w s}}{R_{s}}=\left(\frac{\dot{M}_{w}}{\dot{M}_{a}} \frac{a}{2}\right)^{-\frac{1}{2}} \frac{\sqrt{v_{w}}}{\tilde{M}^{2}} \sim \frac{1}{100}
$$

Note the high Mach number is a result of the fact that in an aspherical bubble the shock creating the shear flow will be oblique and therefore will not strongly decelerate the wind material. In addition the Mach number is an inverse function of the sound speed. The strong cooling in the postshock region keeps this speed low, which also helps to keep the Mach number high. The simulated shear flow has velocity on order Mach 10 (as opposed to the compression Mach number $\tilde{M}=100$ ) and we can couple equation (17) with the result of Cantó and Raga, to find that

$$
\frac{\Delta R_{w s}}{R_{s}} \frac{L_{t}}{\Delta R} \sim 1
$$

This suggests that the flow becomes turbulent only on a size comparable to that of the bubble, and that the focused tangential flow will have time to influence the dynamics before mixing occurs.

Two features this calculation doesn't take into account further reduce the chance that turbulence will dominate the dynamics. First, the continuous supply of fresh wind material along the length of the shear flow is ignored as it was in the Cantó \& Raga estimate. This effect would allow unmixed wind material to be continually resupplied at high latitudes, possibly at a rate faster than the turbulence incorporates material. Second, magnetic fields are ignored. Fields may thread the shell especially when the bubble is small. There is some evidence that toroidal fields, which are present in magnetocentrifugal wind launching models, retard the entrainment of envelope material (Rosen et al. 1999). Poloidal fields at moderate 
Mach numbers can also become wrapped up in vortices in the mixing fluids, strengthening the field which in turn stabilizes the shear flow (Frank et al. 1996).

We have neglected effects in our calculation that may play a mediating role. The most important lie in the crude way in which we treat the geometry of the non-spherical shock. We also haven't considered the early transition from a spherical to an elliptical bubble, when the shear flow Mach number will be more modest. Also, when the shear is stronger, centrifugal forces in the bubble may be important. The details of what instabilities form and how they grow in these geometries could affect the growth rate of the turbulence. Thus while the estimate (18) is suggestive, how quickly the flow becomes turbulent merits further study.

\subsubsection{Direction of the turbulent flow}

Taking the opposite view, we can assume that turbulence will be important dynamically at some time. How then would the resulting mixed fluid behave? Wilkin \& Stahler (1998) assume the postshock flow is dominated by the shocked ambient gas and will be carried down towards the disk. While there is much more mass in the ambient material, the momentum in the wind material is quite high and the flow could be driven poleward. Below we calculate the direction a mixed flow would take in a wind blown bubble shell using a simple model.

In figure 10 we show preshock and postshock regions for the ambient and wind material with a mixing layer sandwiched between. These regions denoted by subscripts $a, w$, and $L$ respectively. We assume the infall $v_{a}$ and wind velocities $v_{w}$ are constant and the turbulent boundary layer grows into the postshock regions at the same constant velocity $v_{0}$. In this framework the question becomes: is $v_{L}$ positive (i.e.poleward) or negative (i.e.equatorward)?

Mass conservation implies:

$$
\rho_{L} 2 v_{0}=\rho_{s a} v_{0}-\rho_{s w}\left(-v_{0}\right) \Rightarrow \rho_{L}=\frac{1}{2}\left(\rho_{s w}+\rho_{s a}\right)
$$

Likewise, momentum conservation for the component moving parallel to the faces of the boundaries implies

$$
\left(\rho_{L} v_{L}\right) 2 v_{0}=\left(\rho_{s a} v_{s a}\right) v_{0}-\left(\rho_{s w} v_{s w}\right)\left(-v_{0}\right) \Rightarrow 2 \rho_{L} v_{L}=\rho_{s a} v_{s a}+\rho_{s w} v_{s w}
$$

The preshock densities are determined from the mass flux and velocity through $\rho=\dot{M} /\left(4 \pi R_{s}^{2} v\right)$, assuming the shell is thin. By taking the shocks to be isothermal, we obtain the postshock densities by multiplying the preshock densities by $\tilde{M}^{2}$ where $\tilde{M}$ is the Mach number. Because 


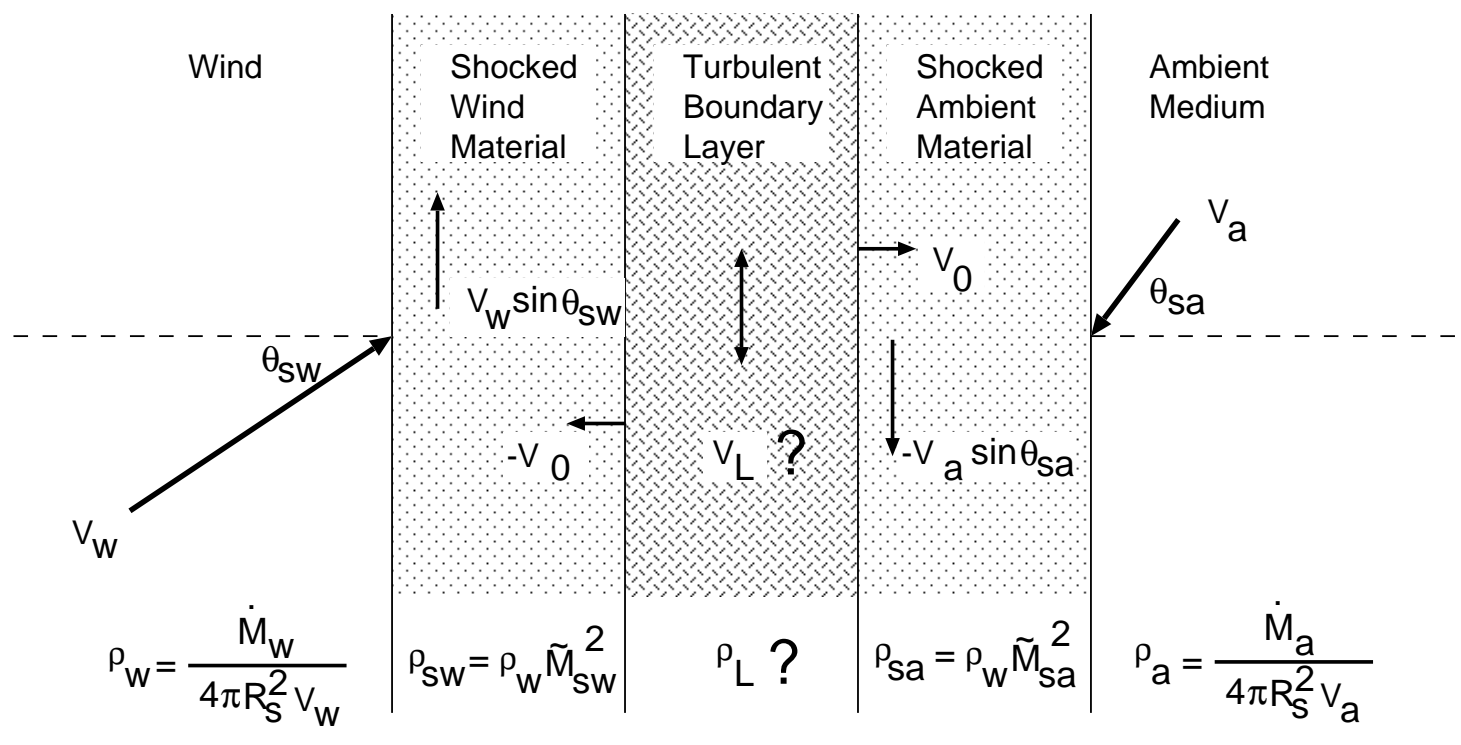

Fig. 10.- Cartoon for a mixing model. The diagram represents a small section of the bubble's shell with wind and ambient material encountering shock layers at an oblique angle. The central mixing layer subsumes material from postshock wind and ambient material which both have velocities parallel to the layer interfaces. The text describes the calculation of the direction of the velocity in the mixing layer. For parameters of interest, we find that the wind determines the direction of the mean velocity of the turbulent region. 
the fluid velocity parallel to the shock remains unchanged through the front, $v_{s a}=-v_{a} \sin \theta_{s a}$, and $v_{s w}=v_{w} \sin \theta_{s w}$. Incorporating these velocities and solving the equations (19) and (20) for $v_{L}$ gives:

$$
v_{L}=\frac{1}{2 \rho_{l}} \frac{\dot{M}_{a} \tilde{M}_{a}^{2}}{4 \pi R_{s}^{2}} \sin \theta_{s a}\left(\frac{\dot{M}_{w}}{\dot{M}_{a}}\left(\frac{\tilde{M}_{s w}}{\tilde{M}_{s a}}\right)^{2} \frac{\sin \theta_{s w}}{\sin \theta_{s a}}-1\right)
$$

We are most interested in whether the mixed flow moves in the direction of the wind or the infall. If the wind and ambient velocities are mostly aligned across the shell, then $\sin \theta_{s a}=\sin \theta_{s w}$, and the condition for the mixed flow moving in the direction of the wind is:

$$
\left(\frac{\dot{M}_{w}}{\dot{M}_{a}}\left(\frac{\tilde{M}_{s w}}{\tilde{M}_{s a}}\right)^{2}\right)>1
$$

For typical values of $\tilde{M}_{s w} \sim 100, \tilde{M}_{s a} \sim 10, \dot{M}_{w} / \dot{M}_{a}=1 / 10$, the left hand side of (22) is $\sim 10$. Thus, the flow moves in the direction of the wind.

We find that the large momentum input of the wind would tend to accelerate the mixed material poleward. At the pole, material collides and sprays ahead of the bubble. This is the Cantó focusing mechanism Cantó \& Rodríguez (1980), called conical converging flows, which operates in simulations under a variety of circumstances (Mellema \& Frank 1997). Material would be cleared and accelerated above the pole leading to a more elongated bubble, and possibly a very narrow jet.

Ignoring shear flows simplifies calculations greatly. We have argued, however, they are important dynamically in bubbles driven into stratified environments whether turbulence is dominant or not. Future models will have to consider such flows in more detail.

\subsection{Properties of young outflows}

As noted in the introduction, Chandler et al. (1996) point to three qualities that models of molecular outflows must reproduce to match their observations of TMC-1 and TMC-1A. They require conical outflow lobes close to the star, evacuated outflow cavities, and moderate $30^{\circ}-40^{\circ}$ opening angles, and they argue that existing models do not explain these effects.

We find, however, that our simulations create outflows meeting the requirements. The opening angle of bubbles in the $f^{\prime}=20$ to $f^{\prime}=50$ cases are $35^{\circ}-40^{\circ}$. To be sure, these 
angles are determined from the density profiles of the simulations and should in the end be determined from synthetic emission maps. However, the angles are not as wide as might naively be expected from a wide angle wind. The similarity to TMC-1, TMC-1A and other young outflows is encouraging because these simulations are on a small spatial and time scale (300 years, $10^{16} \mathrm{~cm}$ ) and would hopefully match up more readily with younger outflows such as these.

The opening angle of the bubble in the $f^{\prime}=10$ case is $60^{\circ}$, which is too wide to compare with TMC-1 and TMC-1A, but wider outflows have been observed. The outflow around IRS1 in Barnard 5 has an opening angle of about $125^{\circ}$ (Velusamy \& Langer 1998). Although these outflows may be explained by multiple non-aligned driving winds or jets, very strong wide angle winds may be at work in such situations.

\subsection{Mass vs. velocity relations.}

Masson \& Chernin (1992) used CO line intensities of NGC 2071, L1551, and HH 46-47 and assumptions about optical depth to obtain the amount of mass per velocity channel in these outflows. They measured a power law in this mass versus velocity relationship of $\Delta m / \Delta v=v^{\gamma}$ with an exponent of $\gamma=-1.8$. With such a steep slope little mass is accelerated to high velocities, leading Chernin and Masson to conclude that any high velocity driver of the molecular outflows must have a small cross-section and consequently cannot be a wide angle wind. Calculations of the observed mass vs. velocity relation in other outflows have been calculated with similar results but with interesting differences. Chandler et al. (1996) have pointed out that it is hard to justify fitting the distributions in their observations with a single power law, although they do try to fit them with two. They find that some "average" power law curve obtains a $\gamma \sim-1.8$. Bally et al. (1999) bring up the issue of sensitivity of the transformation from line intensities to masses to assumptions of optical depth. The universality of a mass vs. velocity relationship may therefore be in question, whether the relationship is even a power law at all, and whether the mass has been properly calculated. We are interested however in making some comparison of models and observations, so it is worth discussing the mass-velocity relation calculated from the simulations.

Figure 11 shows the histograms of ambient mass as a function of projected velocity from our simulations. We able to unambiguously identify the ambient material through the code's fluid tracking and independently by the location of the contact discontinuity. The swept up masses are 1-2 orders of magnitude lower than the masses shown in the TMC1 and TMC1A observations of Chandler et al. (1996). However, our numerical simulations are limited in the 

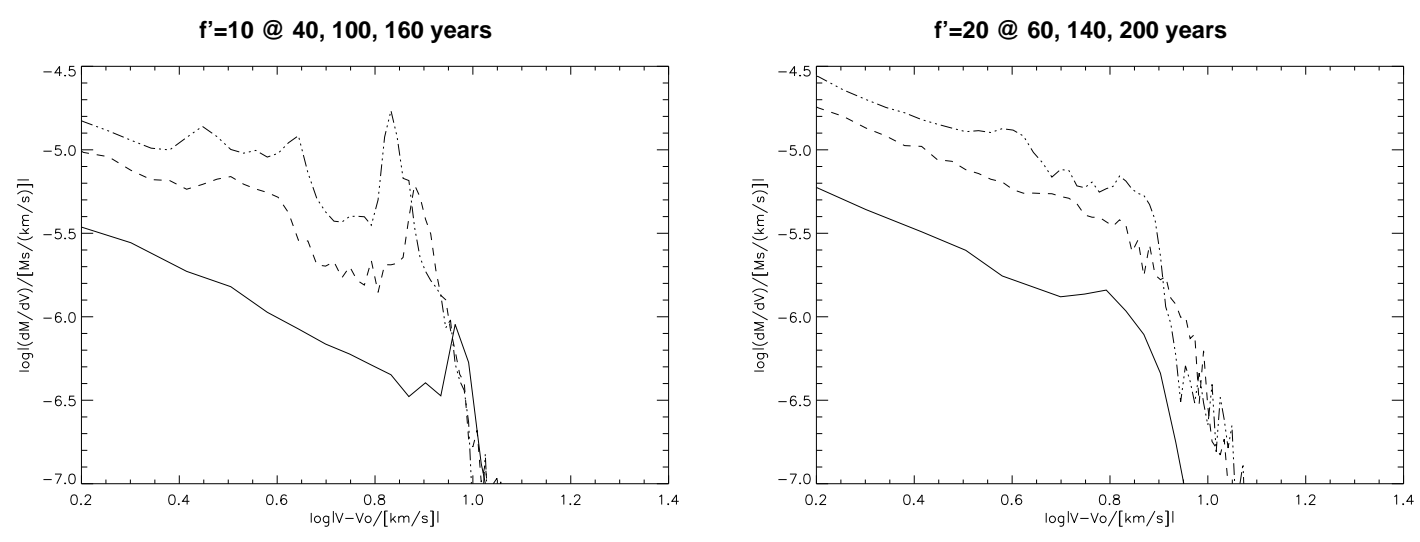

$f^{\prime}=30 @ 80,160,220$ years
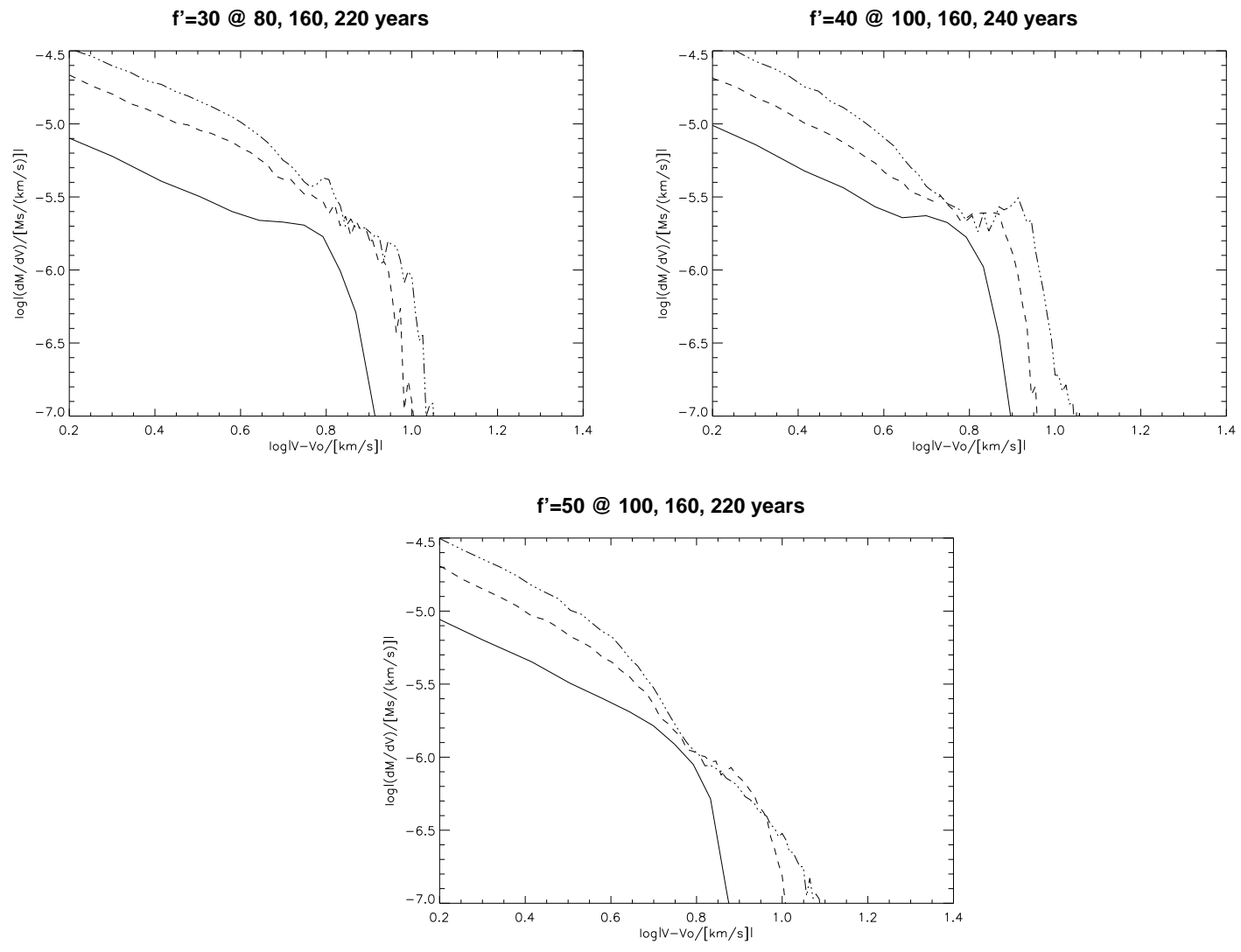

Fig. 11.- Histograms of mass per line-of-sight (LOS) velocity channel for the simulations at different times. As the simulations evolve they sweep up more mass, thus the older curves are above the younger curves. The velocity channels are $0.6 \mathrm{~km} / \mathrm{s}$ wide and the LOS is $45^{\circ}$. 
length of time considered and the spatial region that can be encompassed while maintaining adequate resolution. Thus, our simulations correspond to sweeping up much less envelope material than has occurred in most observed outflow regions. Quantitatively, our models correspond to extremely young, rare systems; qualitatively, many of the effects we show should be present in older objects.

As found in the Chandler et al. (1996) observations the simulation curves are not well fit by a single power law. The curves are logically split into two parts: a "shallow" portion that can be fit on the simulation plots roughly by a power law of exponent ranging from -1.5 to -1.3 and a steeper portion. The shallow portion matches well with the TMC1 and TMC1A results of -1.26 and -0.75 respectively, and is fairly close to the Chernin \& Masson value of $\gamma \sim-1.8$. These fits are subject to some small variation from the choice of where one portion begins and ends, and the size of the velocity bins, but varying among reasonable choices for these only changes the exponents by a tenth or so. The steeper portions of the simulation curves are fit with exponents steeper than -10 , to be compared with an observation of $\sim-3$ (TMC1 and TMC1A) and a jet-model value of -5.6 (Zhang \& Zheng 1997). There appears, however, to be some flattening of the steep portion as the system evolves in the $f^{\prime}=50$ case, which is the most focused model. The slope flattens from -19 to about -7 to -3.25 by 220 years. The focusing accelerates the polar tip and sweeps up increasingly more mass to higher velocities. This acceleration might also occur in other less focused cases when the outflow "breaks out" of the protostar's cloud core into much less dense material. If this breakout flattens the mass vs. velocity curve at high velocity then it could explain the observationally derived curves from older outflows.

The shallow and steep portions of the mass-velocity curves are connected by a "hump," which does not appear in the observationally determined curves. This feature is also seen in the mass-velocity plot of Zhang \& Zheng (1997) and we find that in our simulations it is largely due to the innermost portions of the ambient shock - those farthest from an observer. Optical depth effects could play a role in translating this part of the curve to an accurate integrated CO line profile.

The mass vs. velocity relations for the simulations look promising, with one caveat being that questions remain about this method of matching observations to models. Another caveat is that extrapolating these results to those of large-scale outflows (larger than $0.1 \mathrm{pc}$ ) is not straightforward. Large outflows are likely to involve significantly more material from regions exterior to the cloud than material in the infall in the infall region, and thus the simulations here may refer only to structures on 100 - 1000 AU scales. The Chandler et. al. observations are more relevant in this case. Noting these provisos, the models have mass vs. velocity relations whose shallow portions are about as steep as those quoted by Chernin \& 
Masson, and the steeper portions may flatten as the flow focuses or breaks out of the cloud core. The simulated relations have striking similarities to the observationally derived curves despite the fact that the model systems are driven by wide angle winds.

\section{Conclusion}

We have performed radiative simulations of the interaction of a central spherically symmetric wind with an infalling environment under conditions similar to those found around young stellar objects. We include gravity from the protostar and rotation about the axis. We have examined the effect of varying the relative infall to wind mass flux on the morphology and kinematics of the resulting outflow.

In our simulations, shaping by the toroidal YSO environment of the wind shock, in addition to the outer molecular shock, focuses the outflow, leading to morphologies and kinematics not explored in earlier analytical models. Most importantly, the outflows become progressively more collimated as the central wind becomes weaker relative to the infall. Stronger winds can reverse the inflow of material creating the wide evacuated conical cavities seen in some observations of molecular outflows. Weaker winds are focused more tightly and form elongated, bipolar structures. The simplified snowplow model for these outflows does not explain these variations. Thus, conclusions made from the snowplow model that wide angle winds cannot drive molecular outflows should be reexamined.

We suggest that the effects of turbulent mixing of wind and ambient material, which are not treated explicitly in the simulations, will not significantly alter the results. The mixing is shown to be a relatively inefficient process. The shear speed of the post-shock wind in the simulations is high and we estimate that the poleward focused flow will traverse a large part of the wind blown bubble's circumference before mixing becomes significant. If the mixing does occur, the momentum in the wind is large enough to force the flow towards the poles and possibly form a conical converging flow.

We find a favorable comparison of the mass vs. velocity relations derived from the simulations to those from observations. The shallow portion of the curve at low velocity can be roughly fit by a power law of exponent from about -1.5 to -1 . The steeper portion at high velocity is fit by an exponent of -10 or steeper, although the most focused of our simulations shows a gradual flattening. This leads us to speculate that when the other simulations run to breakout of the cloud core there might be further focusing and a similar transition in the mass vs. velocity curve.

These simulations show that directed outflows can result from wide angle winds. The 
nonlinear interaction of wind and environment leads to dynamics that are not easily modeled outside of simulation, but which lead to behavior similar to what is seen in young outflows. Properties that have been previously been proscribed to jet driven systems can be found in the wide angle winds. Further synthetic observations need to be performed on simulations like those in this paper to indicate the other ways in which the wide angle wind model offers an explanation for molecular outflows.

For low mass YSOs it is probable that wide angle winds will be generated by some form of MHD mechanism associated with an accretion disk (Ouyed \& Pudritz 1997) or disk-star boundary layer (Shu et al. 1994). Although our simulations are hydrodynamic and involve a maximally uncollimated (i.e.isotropic) wind, the shaping of outflows should be general and occur even in the presence of magnetic fields. This is because shock focusing could occur even when the shocks are magnetized. In addition, MHD driven wide angle wind models will likely enhance the outflow collimation in two ways. The first is because these models typically collimate the wind early, in the "wind sphere" region we do not model, to send more momentum along the poles. The second is because toroidal fields are generated and carried out by the wind in such models, providing radially directed hoop stresses in the wind post-shock region.

In future papers we hope to explore in more detail the emissive properties of wide wind driven simulated outflows and make a more direct comparison with observations, as well as study long term behavior. Additionally, these studies of the relative magnitude of the wind have laid the foundation for an examination into how time variation in the wind affects the outflow. With time dependence of the source, which we expect on observational grounds, the outflows can be extremely complicated kinematically. Finally a more detailed exploration of the parameters associated with the asphericity of the wind should be explored allowing a better link between its shape and the resulting properties of the outflows.

We wish to thank Thomas Gardiner for his helpful discussions. This work was supported by NSF Grant AST-0978765 and the University of Rochester's Laboratory for Laser Energetics through a Frank J. Horton Fellowship and their computing facilities.

\section{A. Collapsing Sheet Model}

In this appendix we briefly recapitulate the equations of the sheet density distribution described in Hartmann et al. (1996).

The collapsing sheet is described as a function of spherical radius from the origin $r$ and 
cosine of the polar angle $\mu$. In spherical coordinates the mass density and velocity is:

$$
\begin{aligned}
\frac{\rho}{\rho_{n}}\left(\frac{r}{R_{c}}, \mu\right) & =\left(\operatorname{sech}\left(\eta \mu_{0}\right)\right)^{2}\left(\frac{\eta}{\tanh (\eta)}\right) \frac{\rho_{C M U}}{\rho_{n}}\left(\frac{r}{R_{c}}, \mu\right) \\
\frac{\rho_{C M U}}{\rho_{n}}\left(\frac{r}{R_{c}}, \mu\right) & =\left(\frac{r}{R_{c}}\right)^{-3 / 2}\left(1+\frac{\mu}{\mu_{0}}\right)^{-1 / 2}\left(\frac{\mu}{\mu_{0}}+\frac{2 \mu_{0}}{\frac{r}{R_{c}}}\right)^{-1} \\
\frac{v_{r}}{v_{k}}\left(\frac{r}{R_{c}}, \mu\right) & =-\left(\frac{r}{R_{c}}\right)^{-1 / 2}\left(1+\frac{\mu}{\mu_{0}}\right)^{1 / 2} \\
\frac{v_{\theta}}{v_{k}}\left(\frac{r}{R_{c}}, \mu\right) & =\left(\frac{r}{R_{c}}\right)^{-1 / 2}\left(\mu_{0}-\mu\right)\left(\frac{\mu_{0}+\mu}{\mu_{0}\left(1-\mu^{2}\right)}\right)^{-1 / 2} \\
\frac{v_{\phi}}{v_{k}}\left(\frac{r}{R_{c}}, \mu\right) & =\left(\frac{r}{R_{c}}\right)^{-1 / 2}\left(1-\frac{\mu}{\mu_{0}}\right)^{1 / 2}\left(\frac{1-\mu_{0}^{2}}{1-\mu^{2}}\right)^{1 / 2} .
\end{aligned}
$$

where $\mu_{0}=\mu_{0}\left(r / R_{c}, \mu\right)$ specifies the location (cosine of polar angle) on a reference sphere at some radius $r_{0}$ which a gas parcel at the given coordinates came from. It is defined implicitly by:

$$
\frac{r}{R_{c}}=\frac{1-\mu_{0}^{2}}{1-\frac{\mu}{\mu_{0}}}
$$

The parameter $\eta$ is the ratio of the distance inside-out collapse has progressed into the sheet $r_{0}$, to the scale height $H$ of the initial, static, self-gravitating sheet. It also describes the degree of flattening in the central density distribution. The value of $\eta$ is taken as constant during the simulations. Other basic constants include the centrifugal radius, $R_{c}$, the Keplerian velocity at the centrifugal radius, $v_{k}$, and a density $\rho_{n}$. These in turn can be defined using the mass of the (forming) central star $M_{*}$, and an angular velocity at the radius $r_{0}$ of $\dot{\phi}_{0}$.

$$
\begin{aligned}
R_{c} & =\frac{\dot{\phi}_{0}^{2} r_{0}^{4}}{G M_{*}} \\
v_{k} & =\sqrt{\frac{G M_{*}}{R_{c}}} \\
\rho_{n} & =\frac{\dot{M}_{a}}{4 \pi\left(G M_{*} R_{c}^{3}\right)^{1 / 2}}
\end{aligned}
$$




\section{REFERENCES}

Bachiller, R. 1996, Bipolar molecular outflows from young stars and protostars In $A R A A$, Volume 34, pp. 111-154. Annual Reviews.

Bally, J., Devine, D., Alten, V., \& Sutherland, R. S. 1997, ApJ 478, 603

Bally, J., Reipurth, B., Lada, C. J., \& Billawala, Y. 1999, ApJ 117, 410

Blandford, R. D. \& Payne, D. G. 1982, MNRAS 199, 883

Calvet, N. \& Gullbring, E. 1998, ApJ 509, 802

Cantó, J. \& Raga, A. C. 1991, ApJ 372, 646

Cantó, J. \& Rodríguez, L. F. 1980, ApJ 239, 982

Cassen, P. \& Moosman, A. 1981, Icarus 48, 353

Chandler, C. J., Terebey, S., Barsony, M., Moore, T. J. T., \& Gautier, T. N. 1996, ApJ 471, 308

Chernin, L. M. \& Masson, C. R. 1995, ApJ 455, 182

Cliffe, J. A., Frank, A., Livio, M., \& Jones, T. W. 1995, ApJ 447, L49

Dalgarno, A. \& McCray, R. A. 1972, Heating and ionization of hi regions In L. Goldberg, D. Layzer, \& J. G. Phillips (Eds.), AARA, Volume 10, pp. 375 Annual Reviews Inc.

Frank, A., Jones, T. W., Ryu, D., \& Gaalaas, J. B. 1996, ApJ 460, 777

Frank, A. \& Mellema, G. 1996, ApJ 472, 684

Harten, A. 1983, JCompPhys 49, 357

Hartmann, L., Boss, A., Calvet, N., \& Whitney, B. 1994, ApJ 430, L49

Hartmann, L., Calvet, N., \& Boss, A. 1996, ApJ 464, 387

Hartmann, L. \& Kenyon, S. J. 1996, ARA\&A 34, 207

Königl, A. 1989, ApJ 342, 208

Koo, B.-C. \& McKee, C. F. 1992, ApJ 388, 103

Lepp, S. \& Shull, J. M. 1983, ApJ 270, 578 
Li, Z.-Y. \& Shu, F. H. 1996, ApJ 472, 211

Masson, C. R. \& Chernin, L. M. 1992, ApJ 387, L47

Masson, C. R. \& Chernin, L. M. 1993, ApJ 414, 230

Mellema, G. \& Frank, A. 1997, MNRAS 292, 795

Najita, J. R. \& Shu, F. H. 1994, ApJ 429, 808

Ouyed, R. \& Pudritz, R. E. 1997, ApJ 482, 712

Pudritz, R. E. \& Norman, C. A. 1983, ApJ 274, 677

Raga, A. \& Cabrit, S. 1993, A\&A 278, 267

Raga, A. C., Cabrit, S., \& Cantó, J. 1995, MNRAS 273, 422

Reipurth, B. \& Heathcote, S. 1997, IAU Symposia 182, 3

Rosen, A., Hardee, P., Clarke, D., \& Johnson, A. 1999, ApJ 510, 136

Ryu, D., Brown, G. L., Ostriker, J. P., \& Loeb, A. 1995, ApJ 452, 364-378

Shang, H., Shu, F. H., \& Glassgold, A. E. 1998, ApJ 493, L91

Shu, F., Najita, J., Ostriker, E., Wilkin, F., Ruden, S., \& Lizano, S. 1994, ApJ 429, 781

Shu, F. H. 1992, Gas Dynamics, Volume 2 of The Physics of Astrophysics. University Science Books.

Shu, F. H., Ruden, S. P., Lada, C. J., \& Lizano, S. 1991, ApJ 370, L31

Stahler, S. W. 1994, ApJ 422, 616

Stapelfeldt, K., Burrows, C. J., Krist, J. E., \& Wfpc2 Science Team 1997, IAU Symposia 182, 355

Stone, J. M. \& Norman, M. L. 1994, ApJ 420, 237

Suttner, G., Smith, M. D., Yorke, H. W., \& Zinnecker, H. 1997, A\&A 318, 595

Terebey, S., Shu, F. H., \& Cassen, P. 1984, ApJ 286, 529

Ulrich, R. K. 1976, ApJ 230, 377

Velusamy, T. \& Langer, W. D. 1998, Nat 392, 685 
Wilkin, F. P. \& Stahler, S. W. 1998, ApJ 502, 661

Zhang, Q. \& Zheng, X. 1997, ApJ 474, 719 
This figure "Fig01.png" is available in "png" format from: http://arxiv.org/ps/astro-ph/9910264v1 
This figure "Fig02.png" is available in "png" format from: http://arxiv.org/ps/astro-ph/9910264v1 
This figure "Fig04.png" is available in "png" format from: http://arxiv.org/ps/astro-ph/9910264v1 
This figure "Fig05.png" is available in "png" format from: http://arxiv.org/ps/astro-ph/9910264v1 
This figure "Fig06.png" is available in "png" format from: http://arxiv.org/ps/astro-ph/9910264v1 
This figure "Fig07.png" is available in "png" format from: http://arxiv.org/ps/astro-ph/9910264v1 
This figure "Fig08.png" is available in "png" format from: http://arxiv.org/ps/astro-ph/9910264v1 
This figure "Fig09.png" is available in "png" format from: http://arxiv.org/ps/astro-ph/9910264v1 